\title{
MINIMAL PERIODS OF HOLOMORPHIC MAPS ON COMPLEX TORI
}

\author{
JAUME LLIBRE AND FENG RONG
}

\begin{abstract}
We study the set of minimal periods of holomorphic selfmaps of one and two dimensional complex tori. In particular we characterize when the set of minimal periods of such maps is finite. In fact we have an algorithm for doing this characterization for holomorphic self-maps of an arbitrary dimensional complex tori.
\end{abstract}

\section{Introduction AND Statement of the main RESUlts}

One of the classical invariants in the study of dynamical properties of a map $f$ is the set of minimal periods $\operatorname{Per}(f)$. This set in general is not stable, i.e. it changes if we perturb the map. In particular it is not preserved by a homotopy of the map. It is difficult to analyze the set $\operatorname{Per}(f)$ using tools from algebraic topology. To avoid this difficulty many authors studied the set of homotopy minimal periods, i.e. minimal periods which are preserved by any homotopy (see for instance [1], [10], [7], and [8, Chapt. VI] for an exposition of known results).

On the other hand it is known that holomorphic maps of compact complex manifolds have many periodic points and large sets of minimal periods (see [3], [5] and [12]). Therefore a natural question is: which minimal periods of a holomorphic map $f$ are preserved by a holomorphic homotopy of $f$, and which of them are preserved by any continuous deformation of $f$ ?

In this paper we consider this question for holomorphic self-maps of one- and two-dimensional complex tori, i.e. topologically two- and fourdimensional real tori. The answer is complete when the set of these minimal periods is finite. The same question for holomorphic self-maps of the onedimensional complex torus was partially studied in [12]. Before recalling some of the known results for holomorphic self-maps of complex tori and stating our results, we need to introduce some notation.

Let $X$ be a complex, closed manifold. We define the following sets:

2010 Mathematics Subject Classification. Primary 37C25; Secondary 32H50.

Key words and phrases. Set of minimal periods, periodic points, holomorphic maps on complex tori.

The first author is partially supported by a MCYT/FEDER grant number MTM 200803437, by a CICYT grant number 2009SGR 410 and by ICREA Academia. The second author is partially supported by a NSFC grant number 11001172 and by a CUTECH grant number 20100073120067. 
$\operatorname{Map}(X ; X)$ or simply $\operatorname{Map}(X)$ denotes the set of all continuous self maps of $X$.

$\mathcal{H o l}(X ; X)$ or shortly $\mathcal{H o l}(X)$ denotes the set of all holomorphic self-maps of $X$.

$[X, X]$ denotes the set of all homotopy classes of self-maps of $X$, i.e. $[X, X]=\operatorname{Map}(X) / \sim$, where $\sim$ is the homotopy equivalence relation.

$[X, X]_{\mathcal{H}}$ denotes the set of all homotopy holomorphic classes of holomorphic self-maps of $X$.

For $f, g \in \mathcal{H o l}(X)$ we set $f \approx g$ if there exists a continuous one-parameter family $h_{t} \in \mathcal{H}$ ol $(X)$ for all $t \in[0,1]$ such that $h_{0}=f$ and $h_{1}=g$.

$[X, X]_{\mathcal{H}}=\mathcal{H o l}(X) / \approx$ denotes the set of all homotopy holomorphic classes of holomorphic self-maps of $X$.

Let $f \in \mathcal{H o l}(X)$. We define

$$
\operatorname{HPer}(f)=\bigcap_{g \sim f} \operatorname{Per}(g),
$$

the set of the homotopy minimal periods (shortly homotopy periods) of $f$.

We define

$$
\operatorname{HPer}^{\mathcal{H}}(f)=\bigcap_{\substack{g \sim f \\ g \in \mathcal{H} o l(X)}} \operatorname{Per}(g),
$$

the set of homotopy holomorphic minimal periods (shortly homotopy holomorphic periods) of $f$. We note that here the homotopy is through continuous maps. Of course from the definitions it follows that

$$
\operatorname{HPer}(f) \subset \operatorname{HPer}^{\mathcal{H}}(f),
$$

because the intersection is over a smaller family in the definition of $\operatorname{HPer}^{\mathcal{H}}(f)$.

We remark that in general we have

$$
\operatorname{HPer}(f) \varsubsetneqq \operatorname{HPer}^{\mathcal{H}}(f) .
$$

For explicit examples see [12].

We define

$$
\operatorname{HPer}_{\mathcal{H}}(f)=\bigcap_{\substack{g \approx f \\ g \in \mathcal{H} o l(X)}} \operatorname{Per}(g),
$$

the set of holomorphic homotopic minimal periods (shortly holomorphic homotopy periods) of $f$. We note that here the homotopy is through holomorphic maps. Again from the definitions it follows that

$$
\operatorname{HPer}^{\mathcal{H}}(f) \subset \operatorname{HPer}_{\mathcal{H}}(f) \text {. }
$$

Indeed $f \approx g$ implies that $f \sim g$. Consequently for a given $f \in \mathcal{H}$ ol $(X)$ the intersection in this last definition is over a smaller family than in the previous definition, where the second equality only has meaning if $g \in \mathcal{H o l}(X)$. 
In this paper we shall restrict our space $X$ to be the $r$-dimensional torus $X=\mathbb{T}^{r}$ of complex dimension $r$. Let $f: \mathbb{T}^{r} \rightarrow \mathbb{T}^{r}$ be a holomorphic map. We denote by $A_{f}$ the matrix of the induced homology homomorphism $f_{*}: H_{1}\left(\mathbb{T}^{r}\right) \rightarrow H_{1}\left(\mathbb{T}^{r}\right)$ on the first homological group of $\mathbb{T}^{r}$, which is a $2 r \times 2 r$ integer matrix.

Our first result is for any holomorphic map on $\mathbb{T}^{r}$ with $r$ a positive integer.

Proposition 1. Let $f: \mathbb{T}^{r} \rightarrow \mathbb{T}^{r}$ be a holomorphic map, and $\left[A_{f}\right]$ be the holomorphic map on $\mathbb{T}^{r}$ induced by the integer matrix $A_{f}$ of $f$. Then

$$
\operatorname{Per}(f)=\operatorname{HPer}_{\mathcal{H}}(f)=\operatorname{HPer}^{\mathcal{H}}(f)=\operatorname{HPer}(f)=\operatorname{HPer}\left(\left[A_{f}\right]\right) \text {. }
$$

Proposition 1 for $r=1$ was proved in Theorem 4 and Proposition 5 of [12]. Here it will be proved in section 3 for $r \geq 1$ using similar arguments to the ones of [12].

It is well known (see e.g. [4, Proposition 1.2.3]) that the $2 r \times 2 r$ integer matrix $A_{f}$ has $r$ pairs of conjugate eigenvalues $\alpha_{j} \pm i \beta_{j}$ with $1 \leq j \leq r$ and $\alpha_{j}, \beta_{j} \in \mathbb{R}$. As usual $\mathbb{R}$ denotes the set of real numbers, and $\mathbb{N}$ denotes the set of positive integers.

Theorem 2. Let $f: \mathbb{T}^{1} \rightarrow \mathbb{T}^{1}$ be a holomorphic map, and let $\lambda$ and $\bar{\lambda}$ be the eigenvalues of $A_{f}$. Then $\operatorname{Per}(f)$ is equal to

(E) $\emptyset$ if and only if $\lambda=1$;

(F1) $\{1\}$ if and only if either $\lambda=0$, or $\lambda=-1$, or $\lambda=e^{i 2 \pi / 3}$;

(F2) $\{1,2\}$ if and only if $\lambda=i$;

(F3) $\{1,2,3\}$ if and only if $\lambda=e^{i \pi / 3}$;

(G1) $\mathbb{N} \backslash\{2,3\}$ if and only if $\lambda=-1+i$;

(G2) $\mathbb{N} \backslash\{3\}$ if and only if $\lambda=-\frac{1}{2}+i \frac{\sqrt{7}}{2}$;

(G3) $\mathbb{N} \backslash\{4\}$ if and only if $\lambda=i \sqrt{2}$;

(G4) $\mathbb{N} \backslash\{2\}$ if and only if $\lambda=-2$, or $\lambda=-\frac{3}{2}+i \frac{\sqrt{3}}{2}$;

(G5) $\mathbb{N}$ otherwise.

Theorem 2 is proved in section 2. It improves Theorem 4 of [12] which was not complete.

From Theorem 2 and Proposition 1 we immediately get the following Li-Yorke or S̆arkovskii type result, see [11], [13] and [2].

Corollary 3. Let $f: \mathbb{T}^{1} \rightarrow \mathbb{T}^{1}$ be a holomorphic map. If $f$ has a periodic point of period $n \geq 4$ then $\operatorname{Per}(f)$ is infinite. More precisely $\operatorname{Per}(f) \supseteq$ $\mathbb{N} \backslash\{2,3,4\}$.

As usual $\mathbb{C}$ denotes the set of complex numbers, and $\mathbb{Z}$ denotes the set of integer numbers. We can even partially classify the complex structures of the one-dimensional complex tori that appears in Theorem 2.

Proposition 4. Let $f: \mathbb{T}^{1} \rightarrow \mathbb{T}^{1}$ be a holomorphic map, and let $\lambda$ and $\bar{\lambda}$ be the eigenvalues of $A_{f}$. Suppose $\operatorname{Im} \lambda \neq 0$ and write $\lambda=\alpha+i \beta$, with 
$\alpha, \beta \in \mathbb{R}$ and $\beta>0$. If $\mathbb{T}^{1}=\mathbb{C} / \Lambda$ with $\Lambda=\mathbb{Z}+\tau \mathbb{Z}$, where $\tau$ belongs to the fundamental domain, then we have

(a) if $\beta=1$ then $\tau=i$;

(b) if $\beta=\sqrt{2}$ then $\tau=i \sqrt{2}$;

(c) if $\beta=\frac{\sqrt{3}}{2}$ then $\tau=-\frac{1}{2}+i \frac{\sqrt{3}}{2}$;

(d) if $\beta=\frac{\sqrt{7}}{2}$ then $\tau=-\frac{1}{2}+i \frac{\sqrt{7}}{2}$.

Proposition 4 is proved in section 2. We next extend Theorem 2 and Corollary 3 to holomorphic self-maps of $\mathbb{T}^{2}$.

Theorem 5. Let $f: \mathbb{T}^{2} \rightarrow \mathbb{T}^{2}$ be a holomorphic map, and let $\lambda_{1}, \bar{\lambda}_{1}, \lambda_{2}$ and $\bar{\lambda}_{2}$ be the eigenvalues of $A_{f}$. Then $\operatorname{Per}(f)$ is equal to

(E) $\emptyset$ if and only if $1 \in\left\{\lambda_{1}, \lambda_{2}\right\}$;

(F1) $\{1\}$ if and only if $\left\{\lambda_{1}, \lambda_{2}\right\}$ is either $\{0\}$, or $\{-1\}$, or $\{-1,0\}$, or $\left\{0, e^{i 2 \pi / 3}\right\}$, or $\left\{e^{i 4 \pi / 5}, e^{i 2 \pi / 5}\right\}$, or $\left\{-1, e^{i \pi / 2}\right\}$, or $\left\{-1, e^{i 2 \pi / 3}\right\}$;

(F2) $\{1,2\}$ if and only if $\left\{\lambda_{1}, \lambda_{2}\right\}$ is either $\left\{0, e^{i \pi / 2}\right\}$, or $\left\{e^{i \pi / 2}\right\}$, or $\left\{e^{i 2 \pi / 3}\right.$, $\left.e^{i \pi / 2}\right\}$

(F3) $\{1,3\}$ if and only if $\left\{\lambda_{1}, \lambda_{2}\right\}=\left\{-1, e^{i \pi / 3}\right\}$;

(F4) $\{1,2,3\}$ if and only if $\left\{\lambda_{1}, \lambda_{2}\right\}$ is either $\left\{0, e^{i \pi / 3}\right\}$, or $\left\{e^{i \pi / 2}, e^{i \pi / 3}\right\}$, or $\left\{e^{i \pi / 3}, e^{i 2 \pi / 3}\right\}$;

(F5) $\{1,2,4\}$ if and only if $\left\{\lambda_{1}, \lambda_{2}\right\}=\left\{e^{i \pi / 4}, e^{i 3 \pi / 4}\right\}$;

(F6) $\{1,2,5\}$ if and only if $\left\{\lambda_{1}, \lambda_{2}\right\}=\left\{e^{i \pi / 5}, e^{i 3 \pi / 5}\right\}$;

(F7) $\{1,2,3,6\}$ if and only if $\left\{\lambda_{1}, \lambda_{2}\right\}=\left\{e^{i \pi / 6}, e^{i 5 \pi / 6}\right\}$;

$(\mathrm{G})$ infinite otherwise.

Theorem 5 is proved in section 3. From Theorem 5 and Proposition 1 we immediately get the following Li-Yorke or Sarkovskii type result.

Corollary 6. Let $f: \mathbb{T}^{2} \rightarrow \mathbb{T}^{2}$ be a holomorphic map. If $f$ has a periodic point of period $n \geq 7$ then $\operatorname{Per}(f)$ is infinite.

At the end of section 3, we describe how to extend Theorem 5 and Corollary 6 to holomorphic self-maps of $\mathbb{T}^{r}$ for $r \geq 3$.

\section{HolOMORPHIC MAPS ON THE ONE-DIMENSIONAL COMPLEX TORI}

Let $f: \mathbb{T}^{1} \rightarrow \mathbb{T}^{1}$ be a holomorphic map, and let $\lambda$ and $\bar{\lambda}$ be the eigenvalues of $A_{f}$. Let $\chi(\lambda)=\lambda^{2}-a \lambda+b$ be the characteristic polynomial of $A_{f}$, with $a, b \in \mathbb{Z}$. Set $\lambda=\alpha+i \beta$. Then we have $a=2 \alpha$ and $b=\alpha^{2}+\beta^{2}$.

We first recall the following result from [1] (cf. [7]), which we have reformulated for our purposes.

Theorem 7. Let $f: \mathbb{T}^{1} \rightarrow \mathbb{T}^{1}$ be a continuous map and let $\chi(\lambda)=\lambda^{2}-a \lambda+b$ be the characteristic polynomial of $A_{f}$. Then $\operatorname{HPer}(f)$ is equal to

(E) $\emptyset$ if and only if $1-a+b=0$;

(F1) $\{1\}$ if and only if $(a, b)$ is either $(0,0)$, or $(-1,0)$, or $(-1,1)$, or $(-2,1)$; 
(F2) $\{1,2\}$ if and only if $(a, b)=(0,1)$;

(F3) $\{1,2,3\}$ if and only if $(a, b)=(1,1)$;

(G1) $\mathbb{N} \backslash\{2,3\}$ if and only if $(a, b)=(-2,2)$;

$(\mathrm{G} 2) \mathbb{N} \backslash\{3\}$ if and only if $(a, b)=(-1,2)$;

(G3) $\mathbb{N} \backslash\{4\}$ if and only if $(a, b)=(0,2)$;

(G4) $\mathbb{N} \backslash\{2\}$ if and only if $2+a+b=0$, or $a+b=0$ and $(a, b) \notin\{(0,0)$, $(-1,1),(-2,2)\}$

(G5) $\mathbb{N} \backslash 2 \mathbb{N}$ if and only if $1+a+b=0$ and $(a, b) \notin\{(0,-1),(-1,0)$, $(-2,1)\}$

(G6) $\mathbb{N}$ otherwise.

We now apply Theorem 7 to the holomorphic maps of $\mathbb{T}^{1}$ for proving Theorem 2.

Proof of Theorem 2. Since by Proposition $1 \operatorname{Per}(f)=\operatorname{HPer}(f)$, we shall compute HPer $(f)$ using Theorem 7. So we check the cases in Theorem 7 for $a=2 \alpha$ and $b=\alpha^{2}+\beta^{2}$.

If $1-a+b=0$ then $(1-\alpha)^{2}+\beta^{2}=0$. Thus $(\alpha, \beta)=(1,0)$, which belongs to case $(\mathrm{E})$.

If $b=0$, then $(\alpha, \beta)=(0,0)$, which belongs to case $(\mathrm{F} 1)$.

If $(a, b)=(-2,1)$ then $(\alpha, \beta)=(-1,0)$, which belongs to case $(\mathrm{F} 1)$.

If $(a, b)=(-1,1)$ then $(\alpha, \beta)=\left(-\frac{1}{2}, \pm \frac{\sqrt{3}}{2}\right)$, which belongs to case (F1).

If $(a, b)=(0,1)$ then $(\alpha, \beta)=(0, \pm 1)$, which belongs to case (F2).

If $(a, b)=(1,1)$ then $(\alpha, \beta)=\left(\frac{1}{2}, \pm \frac{\sqrt{3}}{2}\right)$, which belongs to case (F3).

If $(a, b)=(-2,2)$ then $(\alpha, \beta)=(-1, \pm 1)$, which belongs to case (G1).

If $(a, b)=(-1,2)$ then $(\alpha, \beta)=\left(-\frac{1}{2}, \pm \frac{\sqrt{7}}{2}\right)$, which belongs to case (G2).

If $(a, b)=(0,2)$ then $(\alpha, \beta)=(0, \sqrt{2})$, which belongs to case (G3).

If $2+a+b=0$ then $(\alpha+1)^{2}+\beta^{2}+1=0$, which is impossible.

If $a+b=0$ then $(\alpha+1)^{2}+\beta^{2}=1$. Thus $\alpha \in\left\{-2,-\frac{3}{2},-1,-\frac{1}{2}, 0\right\}$ because $-2 \leq \alpha \leq 0$ and $2 \alpha \in \mathbb{Z}$. For $(a, b) \notin\{(0,0),(-1,1),(-2,2)\}$, we then have either $(\alpha, \beta)=(-2,0)$ or $(\alpha, \beta)=\left(-\frac{3}{2}, \pm \frac{\sqrt{3}}{2}\right)$, both belong to case (G4).

If $1+a+b=0$ then $(\alpha+1)^{2}+\beta^{2}=0$. Thus $(\alpha, \beta)=(-1,0)$, which shows that the case (G5) of Theorem 7 cannot occur for a holomorphic map.

Before proving Proposition 4 we recall some basic facts about the complex structures of one-dimensional complex tori.

Let $H$ denote the upper half-plane of $\mathbb{C}$. Then the fundamental domain for the action of the modular group $\Gamma$ on $H$ is given by

$$
\begin{aligned}
D=\left\{z \in H:|z|>1,|\operatorname{Re} z|<\frac{1}{2}\right\} \bigcup & \left\{z \in H:|z| \geq 1, \operatorname{Re} z=-\frac{1}{2}\right\} \\
\bigcup & \left\{z \in H:|z|=1,-\frac{1}{2}<\operatorname{Re} z \leq 0\right\} .
\end{aligned}
$$


For an one-dimensional torus $\mathbb{T}^{1}=\mathbb{C} / \Lambda$, the lattice $\Lambda$ can be chosen to be $\Lambda=\mathbb{Z}+\tau \mathbb{Z}$ with $\tau$ in the fundamental domain.

Proof of Proposition 4. Let $\Pi=(1, \tau)=(1, u+i v)$ be the period matrix of $\mathbb{T}^{1}$. We write

$$
M=\left(\begin{array}{c}
\Pi \\
\bar{\Pi}
\end{array}\right)=\left(\begin{array}{ll}
1 & u+i v \\
1 & u-i v
\end{array}\right), \quad M^{-1}=\frac{i}{2 v}\left(\begin{array}{cc}
u-i v & -u-i v \\
-1 & 1
\end{array}\right) .
$$

We then have

$$
A_{f}=M^{-1}\left(\begin{array}{cc}
\alpha+i \beta & 0 \\
0 & \alpha-i \beta
\end{array}\right) M=\frac{1}{v}\left(\begin{array}{cc}
v \alpha-u \beta & -\left(u^{2}+v^{2}\right) \beta \\
\beta & u \beta+v \alpha
\end{array}\right) .
$$

Since $A_{f}$ is an integral matrix and $\beta \neq 0$, we have

$$
\frac{\beta}{v} \in \mathbb{Z}, \quad 2 u \frac{\beta}{v} \in \mathbb{Z} \text { and }\left(u^{2}+v^{2}\right) \frac{\beta}{v} \in \mathbb{Z} .
$$

If $\beta=1$ then $\frac{1}{v} \in \mathbb{Z}$ implies $v=1$ because $v>\frac{1}{2}$. Thus $\frac{\beta}{v}=1$ and $2 u \in \mathbb{Z}$ implies $u=0$ or $u=-\frac{1}{2}$. But we also have $u^{2}+v^{2} \in \mathbb{Z}$, so $u=0$. This gives (a).

If $\beta=\sqrt{2}$ then $\frac{\sqrt{2}}{v} \in \mathbb{Z}$ implies $v=\sqrt{2}$ because $v>\frac{\sqrt{2}}{2}$. Thus $\frac{\beta}{v}=1$ and $2 u \in \mathbb{Z}$ implies $u=0$ or $u=-\frac{1}{2}$. But we also have $u^{2}+v^{2} \in \mathbb{Z}$, so $u=0$. This gives (b).

If $\beta=\frac{\sqrt{3}}{2}$ then $\frac{\sqrt{3}}{2 v} \in \mathbb{Z}$ implies $v=\frac{\sqrt{3}}{2}$ because $v>\frac{\sqrt{3}}{4}$. Thus $\frac{\beta}{v}=1$ and $2 u \in \mathbb{Z}$ implies $u=-\frac{1}{2}$, which also satisfies $u^{2}+v^{2} \in \mathbb{Z}$. This gives (c).

If $\beta=\frac{\sqrt{7}}{2}$ then $\frac{\sqrt{7}}{2 v} \in \mathbb{Z}$ implies $v=\frac{\sqrt{7}}{2}$ because $v>\frac{\sqrt{7}}{4}$. Thus $\frac{\beta}{v}=1$ and $2 u \in \mathbb{Z}$ implies $u=0$ or $u=-\frac{1}{2}$. But we also have $u^{2}+v^{2} \in \mathbb{Z}$, so $u=-\frac{1}{2}$. This gives $(\mathrm{d})$.

\section{HOLOMORPHIC MAPS ON THE HIGHER-DIMENSIONAL COMPLEX TORI}

Nielsen fixed point theory [9] turns out to be a powerful tool in the study of the set of periods of a map.

As usual the Nielsen number of a map $f$ will be denoted by $N(f)$. For a continuous map $f: \mathbb{T}^{r} \rightarrow \mathbb{T}^{r}$, it is well known that $N(f)=\left|\operatorname{det}\left(I-A_{f}\right)\right|=$ $|\chi(1)|$, where $\chi$ is the characteristic polynomial of $A_{f}$. Note that if $f$ is holomorphic then we have $N(f)=\chi(1)$ because $\chi(1)>0$.

We need the following result from [10].

Theorem 8. Let $f: \mathbb{T}^{r} \rightarrow \mathbb{T}^{r}$ be a continuous map. Then $m \notin \operatorname{HPer}(f)$ if and only if either $N\left(f^{m}\right)=0$ or $N\left(f^{m}\right)=N\left(f^{m / p}\right)$ for some prime factor $p$ of $m$.

Proof of Proposition 1. We always have $\operatorname{HPer}(f)=\operatorname{HPer}\left(\left[A_{f}\right]\right)$ because $f \sim$ $\left[A_{f}\right]$. Therefore it suffices to show that $\operatorname{Per}(f)=\operatorname{HPer}(f)$. Since we always have $\operatorname{Per}(f) \supset \operatorname{HPer}(f)$, we only need to show $\operatorname{Per}(f) \subset \operatorname{HPer}(f)$. 
Assume $n \in \operatorname{Per}(f)$ and let $x \in \mathbb{T}^{r}$ be a point with a minimal period of $n$. Then $x \in \operatorname{Fix}\left(f^{n}\right)$, but $x \notin \operatorname{Fix}\left(f^{n / p}\right)$, where $p$ is a prime factor of $n$. Since $\operatorname{Fix}\left(f^{n / p}\right) \subset \operatorname{Fix}\left(f^{n}\right)$, we then have $\# \operatorname{Fix}\left(f^{n}\right)>\# \operatorname{Fix}\left(f^{n / p}\right)$. Since $f$ is a holomorphic torus map, we have $N\left(f^{m}\right)=\# \operatorname{Fix}\left(f^{m}\right), m \geq 1$ (see [5] or [8, Theorem 4.3.14]). Thus by Theorem 8, we have $n \in \operatorname{HPer}(f)$.

The following result was proved in [1], see also [10].

Theorem 9. Let $f: \mathbb{T}^{r} \rightarrow \mathbb{T}^{r}$ be a continuous map. Then $\operatorname{HPer}(f)$ is of one of the following three (mutually exclusive) types:

(E) $\operatorname{HPer}(f)$ is empty if and only if 1 is an eigenvalue of $A_{f}$;

(F) $\operatorname{HPer}(f)$ in nonempty but finite if and only if all the eigenvalues of $A_{f}$ are either zero or roots of unity not equal to 1 ;

(G) $\operatorname{HPer}(f)$ is infinite.

Now let $f: \mathbb{T}^{2} \rightarrow \mathbb{T}^{2}$ be a holomorphic map, and let $\lambda_{1}, \bar{\lambda}_{1}, \lambda_{2}$ and $\bar{\lambda}_{2}$ be the eigenvalues of $A_{f}$. Let $\chi(\lambda)=\lambda^{4}-a \lambda^{3}+b \lambda^{2}-c \lambda+d$ be the characteristic polynomial of $A_{f}$, with $a, b, c, d \in \mathbb{Z}$. Set $\lambda_{1}=\alpha_{1}+i \beta_{1}$ and $\lambda_{2}=\alpha_{2}+i \beta_{2}$. Then we have

We also have

$$
\begin{aligned}
& a=2\left(\alpha_{1}+\alpha_{2}\right), \\
& b=\alpha_{1}^{2}+\beta_{1}^{2}+\alpha_{2}^{2}+\beta_{2}^{2}+4 \alpha_{1} \alpha_{2}, \\
& c=2\left(\alpha_{1}\left(\alpha_{2}^{2}+\beta_{2}^{2}\right)+\alpha_{2}\left(\alpha_{1}^{2}+\beta_{1}^{2}\right)\right), \\
& d=\left(\alpha_{1}^{2}+\beta_{1}^{2}\right)\left(\alpha_{2}^{2}+\beta_{2}^{2}\right) .
\end{aligned}
$$

$$
N(f)=\chi(1)=\left(1-2 \alpha_{1}+\alpha_{1}^{2}+\beta_{1}^{2}\right)\left(1-2 \alpha_{2}+\alpha_{2}^{2}+\beta_{2}^{2}\right) .
$$

Using Theorems 8 and 9 we shall prove Theorem 5 .

Proof of Theorem 5. By Proposition 1, it suffices to consider $\operatorname{HPer}(f)$. By Theorem 9 , we need to show that if $A_{f}$ has either zero or roots of unity as eigenvalues then it is given by one of the listed eighteen cases.

Case (E) is just case (E) from Theorem 9.

Next assume $\lambda_{1}=0$. If $\lambda_{2}=0$ then $N\left(f^{m}\right)=1$ for all $m \geq 1$, thus $\operatorname{HPer}(f)=\{1\}$ by Theorem 8 . This belongs to case (F1).

If $\lambda_{2} \neq 0$ then $\alpha_{2}^{2}+\beta_{2}^{2}=1$. From $a, b, c, d \in \mathbb{Z}$ we get $2 \alpha_{2} \in \mathbb{Z}$. Therefore $\alpha_{2} \in\left\{-1,-\frac{1}{2}, 0, \frac{1}{2}, 1\right\}$.

If $\alpha_{2}=-1$ then $\beta_{2}=0$ and $N\left(f^{m}\right)$ is periodic as $\{4,0, \cdots\}$. Thus $\operatorname{HPer}(f)=\{1\}$, which belongs to case (F1).

If $\alpha_{2}=-\frac{1}{2}$ then $\beta_{2}= \pm \frac{\sqrt{3}}{2}$ and $N\left(f^{m}\right)=3$ for all $m \geq 1$. Thus $\operatorname{HPer}(f)=\{1\}$, which belongs to case (F1).

If $\alpha_{2}=0$ then $\beta_{2}= \pm 1$ and $N\left(f^{m}\right)$ is periodic as $\{2,4, \cdots\}$. Thus $\operatorname{HPer}(f)=\{1,2\}$, which belongs to case (F2).

If $\alpha_{2}=\frac{1}{2}$ then $\beta_{2}= \pm \frac{\sqrt{3}}{2}$ and $N\left(f^{m}\right)$ is periodic as $\{1,3,4,3,1,0, \cdots\}$. Thus HPer $(f)=\{1,2,3\}$, which belongs to case (F4). 
If $\alpha_{2}=1$ then $\beta_{2}=0$ which belongs to case (E).

Now assume $\lambda_{1} \neq 0$ and $\lambda_{2} \neq 0$. Then $\alpha_{1}^{2}+\beta_{1}^{2}=\alpha_{2}^{2}+\beta_{2}^{2}=1$. From $a, b, c, d \in \mathbb{Z}$ we get $2\left(\alpha_{1}+\alpha_{2}\right) \in \mathbb{Z}$ and $4 \alpha_{1} \alpha_{2} \in \mathbb{Z}$. Set $p=2\left(\alpha_{1}+\alpha_{2}\right)$ and $q=4 \alpha_{1} \alpha_{2}$. Then $\alpha_{1,2}=\frac{p \pm \sqrt{p^{2}-4 q}}{4}$. Since $\alpha_{1,2} \in \mathbb{R}$ and $\left|\alpha_{1,2}\right| \leq 1$, we have $p \in\{-4,-3,-2,-1,0,1,2,3,4\}$ while $\frac{p^{2}-(4-p)^{2}}{4} \leq q \leq \frac{p^{2}}{4}$ for $p \geq 0$ and $\frac{p^{2}-(4+p)^{2}}{4} \leq q \leq \frac{p^{2}}{4}$ for $p<0$.

If $p=4$ then $q=4$. This gives $\left(\alpha_{1}, \beta_{1}\right)=\left(\alpha_{2}, \beta_{2}\right)=(1,0)$, which belongs to case (E).

If $p=3$ then $q=2$. This gives $\left(\alpha_{1}, \beta_{1}\right)=(1,0)$ and $\left(\alpha_{2}, \beta_{2}\right)=\left(\frac{1}{2}, \pm \frac{\sqrt{3}}{2}\right)$, which belongs to case (E).

If $p=2$ then $q \in\{1,0\}$. If $q=0$ then $\left(\alpha_{1}, \beta_{1}\right)=(1,0)$ and $\left(\alpha_{2}, \beta_{2}\right)=$ $(0, \pm 1)$, which belongs to case $(\mathrm{E})$.

If $q=1$ then $\left(\alpha_{1}, \beta_{1}\right)=\left(\frac{2+\sqrt{3}}{4}, \pm \frac{\sqrt{9-4 \sqrt{3}}}{4}\right),\left(\alpha_{2}, \beta_{2}\right)=\left(\frac{2-\sqrt{3}}{4}, \pm \frac{\sqrt{9+4 \sqrt{3}}}{4}\right)$ and one readily checks that $\alpha_{1}^{\prime}=\operatorname{Re} \lambda_{1}^{2}=\frac{-1+4 \sqrt{3}}{8}$ and $\alpha_{2}^{\prime}=\operatorname{Re} \lambda_{2}^{2}=\frac{-1-4 \sqrt{3}}{8}$. This implies $\operatorname{Tr} A^{2}=2\left(\alpha_{1}^{\prime}+\alpha_{2}^{\prime}\right)=-\frac{1}{2} \notin \mathbb{Z}$, which is impossible.

If $p=1$ then $q \in\{0,-1,-2\}$. If $q=-2$ then $\left(\alpha_{1}, \beta_{1}\right)=(1,0)$ and $\left(\alpha_{2}, \beta_{2}\right)=\left(-\frac{1}{2}, \pm \frac{\sqrt{3}}{2}\right)$, which belongs to case $(\mathrm{E})$.

If $q=0$ then $\left(\alpha_{1}, \beta_{1}\right)=\left(\frac{1}{2}, \pm \frac{\sqrt{3}}{2}\right)$ and $\left(\alpha_{2}, \beta_{2}\right)=(0, \pm 1)$. We get $N\left(f^{m}\right)$ is periodic as $\{2,12,8,12,2,0, \cdots\}$. Thus $\operatorname{HPer}(f)=\{1,2,3\}$, which belongs to case $(\mathrm{F} 4)$.

If $q=-1$ then $\left(\alpha_{1}, \beta_{1}\right)=\left(\frac{1+\sqrt{5}}{4}, \pm \frac{\sqrt{10-2 \sqrt{5}}}{4}\right)$ and $\left(\alpha_{2}, \beta_{2}\right)=\left(\frac{1-\sqrt{5}}{4}\right.$, $\left.\pm \frac{\sqrt{10+2 \sqrt{5}}}{4}\right)$. We get $N\left(f^{m}\right)$ is periodic as $\{1,5,1,5,16,5,1,5,1,0, \cdots\}$. Thus HPer $(f)=\{1,2,5\}$, which belongs to case (F6).

If $p=0$ then $q \in\{0,-1,-2,-3,-4\}$. If $q=-4$ then $\left(\alpha_{1}, \beta_{1}\right)=(1,0)$ and $\left(\alpha_{2}, \beta_{2}\right)=(-1,0)$, which belongs to case $(\mathrm{E})$.

If $q=0$ then $\left(\alpha_{1}, \beta_{1}\right)=(0, \pm 1)$ and $\left(\alpha_{2}, \beta_{2}\right)=(0, \pm 1)$. We get $N\left(f^{m}\right)$ is periodic as $\{4,16, \cdots\}$. Thus $\operatorname{HPer}(f)=\{1,2\}$, which belongs to case (F2).

If $q=-1$ then $\left(\alpha_{1}, \beta_{1}\right)=\left(\frac{1}{2}, \pm \frac{\sqrt{3}}{2}\right)$ and $\left(\alpha_{2}, \beta_{2}\right)=\left(-\frac{1}{2}, \pm \frac{\sqrt{3}}{2}\right)$. We get $N\left(f^{m}\right)$ is periodic as $\{3,9,12,9,3,0, \cdots\}$. Thus $\operatorname{HPer}(f)=\{1,2,3\}$, which belongs to case (F4).

If $q=-2$ then $\left(\alpha_{1}, \beta_{1}\right)=\left(\frac{\sqrt{2}}{2}, \pm \frac{\sqrt{2}}{2}\right)$ and $\left(\alpha_{2}, \beta_{2}\right)=\left(-\frac{\sqrt{2}}{2}, \pm \frac{\sqrt{2}}{2}\right)$. We get $N\left(f^{m}\right)$ is periodic as $\{2,4,2,16,2,4,2,0, \cdots\}$. Thus $\operatorname{HPer}(f)=\{1,2,4\}$, which belongs to case (F5).

If $q=-3$ then $\left(\alpha_{1}, \beta_{1}\right)=\left(\frac{\sqrt{3}}{2}, \pm \frac{1}{2}\right)$ and $\left(\alpha_{2}, \beta_{2}\right)=\left(-\frac{\sqrt{3}}{2}, \pm \frac{1}{2}\right)$. We get $N\left(f^{m}\right)$ is periodic as $\{1,3,4,3,1,16,1,3,4,3,1,0, \cdots\}$. Thus $\operatorname{HPer}(f)=$ $\{1,2,3,6\}$, which belongs to case (F7).

If $p=-1$ then $q \in\{0,-1,-2\}$. 
If $q=-1$ then $\left(\alpha_{1}, \beta_{1}\right)=\left(\frac{-1-\sqrt{5}}{4}, \pm \frac{\sqrt{10-2 \sqrt{5}}}{4}\right)$ and $\left(\alpha_{2}, \beta_{2}\right)=\left(\frac{-1+\sqrt{5}}{4}\right.$, $\left.\pm \frac{\sqrt{10+2 \sqrt{5}}}{4}\right)$. We get $N\left(f^{m}\right)$ is periodic as $\{5,5,5,5,0, \cdots\}$. Thus $\operatorname{HPer}(f)=$ $\{1\}$, which belongs to case (F1).

If $q=0$ then $\left(\alpha_{1}, \beta_{1}\right)=\left(-\frac{1}{2}, \pm \frac{\sqrt{3}}{2}\right)$ and $\left(\alpha_{2}, \beta_{2}\right)=(0, \pm 1)$. We get $N\left(f^{m}\right)$ is periodic as $\{6,12, \cdots\}$. Thus $\operatorname{HPer}(f)=\{1,2\}$, which belongs to case (F2).

If $q=-2$ then $\left(\alpha_{1}, \beta_{1}\right)=(-1,0)$ and $\left(\alpha_{2}, \beta_{2}\right)=\left(\frac{1}{2}, \pm \frac{\sqrt{3}}{2}\right)$. We get $N\left(f^{m}\right)$ is periodic as $\{4,0,16,0,4,0, \cdots\}$. Thus $\operatorname{HPer}(f)=\{1,3\}$, which belongs to case (F3).

If $p=-2$ then $q \in\{1,0\}$.

If $q=1$ then $\left(\alpha_{1}, \beta_{1}\right)=\left(\frac{-2-\sqrt{3}}{4}, \pm \frac{\sqrt{9-4 \sqrt{3}}}{4}\right),\left(\alpha_{2}, \beta_{2}\right)=\left(\frac{-2+\sqrt{3}}{4}, \pm \frac{\sqrt{9+4 \sqrt{3}}}{4}\right)$ and a similar argument as above shows that this is impossible.

If $q=0$ then $\left(\alpha_{1}, \beta_{1}\right)=(-1,0)$ and $\left(\alpha_{2}, \beta_{2}\right)=(0, \pm 1)$. We get $N\left(f^{m}\right)$ is periodic as $\{8,0, \cdots\}$. Thus $\operatorname{HPer}(f)=\{1\}$, which belongs to case (F1).

If $p=-3$ then $q=2$. This gives $\left(\alpha_{1}, \beta_{1}\right)=(-1,0)$ and $\left(\alpha_{2}, \beta_{2}\right)=\left(-\frac{1}{2}\right.$, $\left.\pm \frac{\sqrt{3}}{2}\right)$. We get $N\left(f^{m}\right)$ is periodic as $\{12,0, \cdots\}$. Thus $\operatorname{HPer}(f)=\{1\}$, which belongs to case (F1).

If $p=-4$ then $q=4$. This gives $\left(\alpha_{1}, \beta_{1}\right)=\left(\alpha_{2}, \beta_{2}\right)=(-1,0)$. We get $N\left(f^{m}\right)$ is periodic as $\{16,0, \cdots\}$. Thus $\operatorname{HPer}(f)=\{1\}$, which belongs to case (F1).

It is evident from the above proof that similar arguments will also give us classifications of when the set of minimal periods of holomorphic self-maps of $r$-dimensional complex tori is finite, for any $r \geq 3$. We briefly outline the algorithm below. Note however that the computation involved will get more complicated.

Let $f: \mathbb{T}^{r} \rightarrow \mathbb{T}^{r}$ be a holomorphic map and let $\lambda_{j}, \bar{\lambda}_{j}, 1 \leq j \leq r$, be the eigenvalues of $A_{f}$. Set $\lambda_{j}=\alpha_{j}+i \beta_{j}$. Then the characteristic polynomial of $A_{f}$, with integral coefficients, is given by $\chi(\lambda)=\prod_{j=1}^{r}\left(\lambda-\lambda_{j}\right)\left(\lambda-\bar{\lambda}_{j}\right)$ and the Nielsen number of $f$ is given by $N(f)=\chi(1)=\prod_{j=1}^{r}\left(1-2 \alpha_{j}+\alpha_{j}^{2}+\beta_{j}^{2}\right)$.

By Proposition 1, we have $\operatorname{Per}(f)=\operatorname{HPer}(f)$. We can then use Theorems 8 and 9 to classify when $\operatorname{HPer}(f)$, thus $\operatorname{Per}(f)$, is finite.

By Theorem 9 case (E), we have $\operatorname{Per}(f)=\emptyset$ if and only if $\lambda_{j}=1$ for some $j$. By Theorem 9 case $(\mathrm{F})$, we then assume that all $\lambda_{j}$ are either zero or roots of unity not equal to 1 .

If some $\lambda_{j}$ is zero, say $\lambda_{r}=0$, then $\chi(\lambda)=\lambda^{2} \prod_{j=1}^{r-1}\left(\lambda-\lambda_{j}\right)\left(\lambda-\bar{\lambda}_{j}\right)$. Note that $\tilde{\chi}(\lambda)=\prod_{j=1}^{r-1}\left(\lambda-\lambda_{j}\right)\left(\lambda-\bar{\lambda}_{j}\right)$ is still a polynomial with integral coefficients. Thus the classification for this case is given by the $(r-1)-$ dimensional classification. 
We can then assume that $\alpha_{j}^{2}+\beta_{j}^{2}=1$ and $\alpha_{j} \neq 1$ for all $j$. Note that this automatically implies that all $\lambda_{j}$ are roots of unity (see e.g. [6, $\S 34$, Lemma (a)]), and thus $N\left(f^{m}\right)$ is periodic. The classification for this case is then given by studying the constrains posted by the integral coefficients of $\chi(\lambda)$, such as $\operatorname{Tr} A_{f}=2 \sum_{j=1}^{r} \alpha_{j} \in \mathbb{Z}$.

The above consideration gives us finitely many cases when $\operatorname{Per}(f)$ is finite. Thus we have the following Li-Yorke or Sarkovskii type result.

Proposition 10. Let $f: \mathbb{T}^{r} \rightarrow \mathbb{T}^{r}$ be a holomorphic map. There exists a finite integer $l(r)$ such that if $f$ has a periodic point of period $n \geq l(r)$ then $\operatorname{Per}(f)$ is infinite.

From Corollaries 3 and 6 we know that $l(1)=4$ and $l(2)=7$. And by the discussion above, it is clear that $l(r)$ is non-decreasing. It is natural to conjecture that $l(r)$ is actually increasing and it will be interesting to see whether one can express $l(r)$ in terms of $r$.

\section{REFERENCES}

[1] L. Alsedì, S. Baldwin, J. Llibre, R. Swanson And W. Szlenk, Minimal sets of periods for torus maps via Nielsen numbers, Pacific J. Math. 169 (1995), 1-32.

[2] L. Alsedè, J. Llibre And M. Misiurewicz, Combinatorial Dynamics and Entropy in Dimension One, (Second Edition), Advanced Series in Nonlinear Dynamics, Vol. 5, World Scientific, Singapore, 2000.

[3] A. Beardon, Iteration of Rational Functions, Graduate Texts in Math., Vol. 132, Springer-Verlag, New York, 1991.

[4] C. Birkenhake and H. Lange, Complex Abelian Varieties, (Second Edition), Springer-Verlag, Berlin Heidelberg, 2004.

[5] N. Fagella And J. Llibre, Periodic points of holomorphic maps via Lefschetz numbers, Trans. Amer. Math. Soc. 352 (2000), 4711-4730.

[6] E. Hecke, Lectures on the Theory of Algebraic Numbers, Graduate Texts in Math., Vol. 77, Springer-Verlag, New York, 1981.

[7] J. Jezierski and W. Marzantowicz, Homotopy minimal periods for maps of three dimensional nilmanifolds, Pacific J. Math. 209 (2003), 85-101.

[8] J. Jezierski and W. Marzantowicz, Homotopy Methods in Topological Fixed and Periodic Points Theory, Topological Fixed Point Theory and Its Applications 3, Springer, Dordrecht, 2006.

[9] B. JiAng, Lectures on Nielsen Fixed Point Theory, Contemp. Math. 14, Amer. Math. Soc., Providence, R.I., 1983.

[10] B. Jiang And J. Llibre, Minimal sets of periods for torus maps, Discrete Contin. Dyn. Syst. 4 (1998), 301-320.

[11] T.Y. Li And J. Yorke, Period three implies chaos, Amer. Math. Monthly 82 (1975), 985-992.

[12] J. Llibre And W. Marzantowicz, Homotopy minimal periods of holomorphic maps on surfaces, Funct. Approx. Comment. Math. 40 (2009), 309-326.

[13] A.N. S̆ARKovSkiI, Co-existence of the cycles of a continuous map of the line into itself, (in Russian), Ukrain. Math. Zh. 16(1) (1964), 61-71.

Departament de Matemàtiques, Universitat Autònoma de Barcelona, 08193

Bellaterra, Barcelona, Catalonia, Spain

E-mail address: jllibre@mat.uab.cat 
Department of Mathematics, Shanghai Jiao Tong University, 800 Dong Chuan Road, Shanghai, 200240, P.R. China

E-mail address: frong@sjtu.edu.cn 